\title{
Automated guided vehicle mission reliability modelling using a combined fault tree and Petri net approach
}

\author{
Rundong Yan $^{1}$ • Lisa M. Jackson ${ }^{1} \cdot$ Sarah J. Dunnett ${ }^{1}$
}

Received: 30 November 2016 / Accepted: 16 February 2017 / Published online: 21 March 2017

(C) The Author(s) 2017. This article is published with open access at Springerlink.com

\begin{abstract}
Automated guided vehicles (AGVs) are being extensively used for intelligent transportation and distribution of materials in warehouses and autoproduction lines due to their attributes of high efficiency and low costs. Such vehicles travel along a predefined route to deliver desired tasks without the supervision of an operator. Much effort in this area has focused primarily on route optimisation and traffic management of these AGVs. However, the health management of these vehicles and their optimal mission configuration have received little attention. To assure their added value, taking a typical AGV transport system as an example, the capability to evaluate reliability issues in AGVs are investigated in this paper. Following a failure modes effects and criticality analysis (FMECA), the reliability of the AGV system is analysed via fault tree analysis (FTA) and the vehicles mission reliability is evaluated using the Petri net (PN) method. By performing the analysis, the acceptability of failure of the mission can be analysed, and hence the service capability and potential profit of the AGV system can be reviewed and the mission altered where performance is unacceptable. The PN method could easily be extended to have the capability to deal with fleet AGV mission reliability assessment.
\end{abstract}

Rundong Yan

r.yan@lboro.ac.uk

Lisa M. Jackson

1.m.jackson@lboro.ac.uk

Sarah J. Dunnett

s.j.dunnett@lboro.ac.uk

1 Loughborough University, Loughborough, Leicestershire, LE11 3TU, UK
Keywords Automated guided vehicles · Reliability · Petri nets · Fault tree analysis

\section{Introduction}

For intelligent transportation and distribution of materials in warehouses and/or manufacturing facilities, there has been in recent years the increasing use of automated guided vehicles (AGVs). Such vehicles travel along predefined routes to deliver various tasks without the supervision of an onboard operator. As the AGV systems are getting larger and more complex, increasing the efficiency and lowering the operation cost of the AGV system have naturally become the first priorities, via investigating the design and control aspects of the AGV [1-4], by identifying new flow-path layouts including workstation layouts [5-7] and developing advanced traffic management strategies, including vehicle routing and task assignment [8-13]. Trenkle [14] introduced the safety requirements and safety functions for a decentralised controlled AGV system. Three major hazards, i.e. collision with a person, tilting over and falling down, were identified. The effects of the speed of AGVs, the braking distance and detection area requirements as well as the mean time to dangerous failure and performance were analysed. Regarding failure response, Ebben developed a method for failure control management for a special case study of AGVs, an underground transportation system (with loaded and unloaded AGVs considered) [15]. In the area of the reliability modelling of AGVs, Fazlollahtabar [16] created a model to maximise the reliability of AGVs and minimise their repair cost, and Tavana and Fazlollahtabar modelled the reliability of AGVs as a cost function to optimise time and cost objectives [17]. However, fully understanding how AGVs can fail and the causes of such failures is still needed. 
Some progress was made in this area by Duran et al. [18] who attempted to identify the basic failure modes of the light detection and ranging (LIDAR) system and the camera-based computer vision system (CV) on AGVs by using a combined approach of fault tree analysis (FTA) and Bayesian belief networks (BN). In the work, human injury, property damage and vehicle damage were defined as the top events in the fault tree. However, the research did not cover all components and subassemblies included in AGVs.

Modelling using Petri net (PN) has been becoming a common tool and popular research topic to evaluate reliability of a system or a mission. For example, Wu proposed an extended object-oriented Petri net model to analyse the reliability of a phased mission with common cause failures in 2015. [19] On the other hand, from the aspect of industrial applications, Le and Andrews [20] presented a wind turbine asset model to study the degradation, maintenance and inspection processes of different wing tunnel components based on PNs. However, to date the Petri net (PN) method has only been used as a mathematical tool to investigate route planning and control strategies for AGV systems. For example, Luo and $\mathrm{Ni}$ [21] designed a programmable logical controller (PLC) using Petri nets to prevent collisions of vehicles in an AGV system and Nishi and Maeno [22] proposed an approach to optimise the routing planning for AGVs in semiconductor fabrication bays.

However, to the best of the authors knowledge, PNs have rarely been applied to the study of the reliability of AGVs. In particular, their application to mission reliability. This is the aim of the work presented in this paper. In contrast to the combined approach of FTA and BN adopted in [18], the combined use of FTA and PNs adopted here enables not only the analysis of all failure modes of all the subsystems but also an analysis of the mission of the AGV . In addition, the PNs can be easily modified if the mission changed.

In a system with only a few AGVs, the failure of any one of the AGVs will not cause a significant traffic congestion issue. Moreover, the failed AGV can be quickly replaced by back up ones. Hence, such a small scale AGV application system can be easily managed [1]. However, given the increasing number of large scale AGV application systems where a significant number of AGVs share the limited number of travel routes, the failure of any one of these AGVs will cause serious traffic chaos. Hence, for this reason, considering a complete investigation of the reliability issues of all AGV components and subassemblies is important not only to ensure the high reliability and availability of AGVs and their success of delivering prescribed tasks but also to optimise their maintenance strategies and minimise traffic chaos. The reliability issues of the whole AGV system are investigated through assessing the reliability of a typical AGV transport system in this paper, where the capability to consider mission analysis of AGVs is shown. The novelties of this paper can be summarise as the following: an effective reliability assessment of AGVs using combined FTA and PNs has been proposed. Using the techniques developed, the critical phases in the AGV mission can be identified and their failure probability can be obtained. The PN simulation is found to be an efficient and adaptable method to analyse the reliability of complex AGV systems undertaking various tasks.

The remaining part of the paper is organised as follows. In Section 2, the reliability modelling methods are discussed with the AGV application system being the focus of Section 3. Section 4 covers the AGV system reliability model generation and the AGV mission analysis is covered in Section 5. The simulation method adopted and the results are presented in Section 6 with the conclusions in the final section.

\section{Reliability modelling}

One of the most commonly used reliability methods, widely adopted in industrial practise, is fault tree analysis (FTA). This method allows a system failure mode to be expressed in terms of the interactions of its components. Moreover, with the aid of FTA, the probability of system or mission failure can be computed via Boolean logic calculations. This method has been adopted to evaluate the subsystem level failures for the AGV system.

When the system is large and complex, or the mission performed is made up of many phases, FTA can become inaccurate and computationally expensive. In such cases, alternative reliability modelling methods may be better suited to performance analysis, one such technique being Petri nets (PNs), developed by Petri [23]. Similar to FTA, PNs provide an intuitive graphical representation of the system being modelled allowing for reliability investigation. The PN method is a direct bipartite graph which consists of four types of symbol: circles, rectangles, arrows and tokens. Circles represent the places, which are conditions or states such as mission failure, phase failure or component failure; rectangles represent the transitions, more abstractly actions or events which cause the change of condition or state. It should be mentioned that if the time for completing the transition is zero, the rectangle is filled in, otherwise it is hollow; arrows represent arcs which are connections between places and transitions. Arcs with a slash on and a number, $n$, next to the slash represent a combination of $\mathrm{n}$ single arcs and the arc is said to have a weight $n$. No slash always means that the weight is one; and small filled in circles represent tokens which carry the information in the PNs. The tokens move via transitions as long as the enabling condition explained below is satisfied, which gives the dynamic properties of the PNs. The marking of a net at any particular time gives the state of the system being modelled at that time. 
Figure 1 shows an example net where movement of tokens have occurred after a time period, hence the tokens make a transition through the net. The net has two input places (represented by circles) and one output place (drawn as a circle) connected by a timed transition (hollow rectangle) with a time delay $t$. There is one token and three tokens (represented by small filled in circles) in each of the two input places. The input places have arcs with weights 1 and 2 , respectively. Currently, there are no tokens in the output place; however, a transition will be enabled when the number of tokens contained in every input place is equal to or more than the corresponding arc weights. Given this is true for the net shown in Fig. 1, the transition is enabled and the number of tokens equivalent to the output arc number are transferred to the output place; in this instance, one token appears in the output place. The number of tokens moved to the output place is dependent on the corresponding arc weight, hence if the arc weight is ' $n$ ', then $n$ more tokens will appear in the output place after the transition fires.

Petri nets for system representations are built up using these same components. Research has shown the application of these nets to systems that undertake phased missions, where a net is generated for the system and an additional net is developed for the phase, known as a system and phase net, respectively [24]. Extensions for more complex systems have included using three distinct PNs, i.e. phase PN, component PN and master PN [25]. These three kinds of PNs are linked together and interact with each other. Such an extended approach is adopted in this paper to assess the reliability of AGV systems.

\section{Application AGV system and mission}

A typical AGV transport system used in a warehouse for material distribution is chosen for analysis in this research. As shown in Fig. 2, the AGV system consists of various subsystems, where the software control system is central to the AGV's operation. This subsystem is responsible for processing and interpreting the information received from both the laser navigation system and safety system, and sends either motion or operation orders. Linked to this subsystem, there are a number of inputs and outputs. The laser navigation system and the safety system both feed into the control unit.

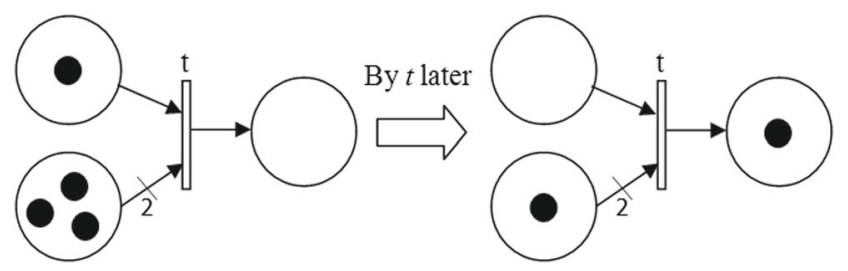

Fig. 1 Petri net model with transitions

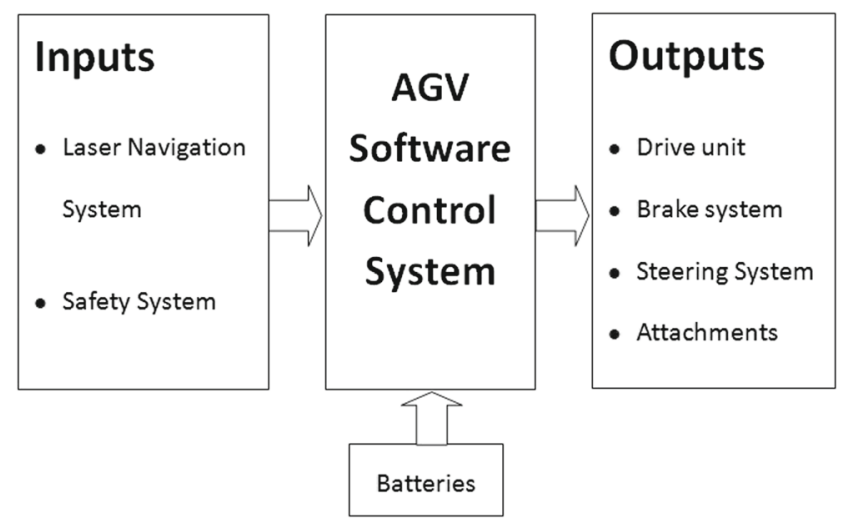

Fig. 2 Typical AGV system

The laser navigation system, like that developed by MacLeod [26], is in essence a position measurement system that is responsible for locating the AGV. The safety system is a collision/avoidance system designed to avoid obstacles that could appear on the pathway with the aid of a laser detection system installed on the AGV. In order to perform its tasks, the AGV has a number of output systems: the drive unit, the brake system, the steering system and the attachments (for lifting, etc.). In terms of the motion or operation orders, these are executed by the drive unit, the brake system and/or the steering system. The drive unit is typically a brushless DC electric motor which is responsible for providing power for the motion and operation of the AGV, the braking system is responsible for slowing down or stopping the AGV and is always applied when the AGV is stationary. The steering system is responsible for manoeuvring the AGV. Attachments refer to additional components that are used to assist moving and carrying of items. All functions require a power system, which is typically lead-acid battery which is responsible for supplying power to the whole AGV system.

In terms of the mission of the AGV, this can be broken up into distinct tasks. First of all, the AGV has to optimise the routes for completing the whole mission, given its assignment. Once in motion, the AGV will travel to the material collection port along the optimised route to pick up the materials. After the AGV is loaded with the materials, it

Table 1 Assumed phase lengths

\begin{tabular}{ll}
\hline Phase & Phase length (h) \\
\hline Mission allocation \& route optimisation & 0.02 \\
Dispatch to station & 0.20 \\
Loading of item & 0.02 \\
Travelling to storage & 0.15 \\
Unloading & 0.02 \\
Travelling back to base & 0.10 \\
\hline
\end{tabular}


will travel to the destination and unload the materials. After successfully distributing the materials, the AGV will travel back to its original parking position. Therefore, the whole mission can be divided into six phases in total, namely (1) mission allocation and route optimisation, (2) dispatch to station, (3) loading of item, (4) travelling to storage, (5) unloading and (6) travelling back to base. The mission can be regarded as successful only when the $\mathrm{AGV}$ is able to operate successfully throughout all these six phases without any break due to component and/or subsystem failures and maintenance. Such a period is named as a maintenance-free operational period (MFOP) [27].

In order to calculate the phase mission reliability, the length of each phase (i.e. the time duration for completing each phase) is required and the values assumed in this work are shown in Table 1 . These values taken are for demonstration purposes, where the total time duration to complete the whole mission is $0.51 \mathrm{~h}$. It is worth mentioning that the assumed values in Table 1 are based on the consultation with an AGV operator. These values would be different for different applications. Therefore, the time duration should be modified correspondingly when considering different AGV applications.

\section{AGV reliability model generation}

\subsection{Subsystem level reliability models}

Initially, a detailed FMECA analysis of the AGV (with an extract illustrated in Table 2) was performed in order to obtain a detailed understanding of the vehicle. Eight subsystems were identified for analysis. In Table 2, the subsystem, the laser navigation system (LNS), is shown where columns labelled S, F, D and RPN refer to the severity ranking, frequency ranking, detectability ranking (all with ratings 1-5) and risk priority number, respectively (calculated as the multiplicity of all three rankings). Similar tables were developed for all AGV subsystems.

The understanding gained from the FMECA was then used to construct fault trees describing the failure of each subsystem.

As an example the failure of the laser navigation system (LNS) is shown in Fig. 3. This subsystem failure can be broken down to be caused by four basic events: laser emitter failure; laser sensor failure; GPS failure and signal transmitter failure. In total, eight subsystem level fault trees have been constructed, varying in size from just 1 gate and 5 events to 3 gates and 11 events. Following this understanding of the interrelationships between failures within the AGV system, this information can be used to establish the likelihood/frequency of AGV subsystem failure. A quantitative analysis of the fault trees has yielded the frequency of failure of each subsystem as shown in Table 3. The data used for the basic events in the subsystem fault trees was based on RAC FMD-97 [28] and expert knowledge.

\subsection{Mission phase reliability models}

Given the AGV undertakes a mission comprised of different phases, the relationship between the subsystems that operate within each phase needs to be understood. Failure models, using fault tree analysis, of these phases within the mission have been developed in this paper and analysed. As the subsystems within the AGV are independent, to reduce the size of the phase fault trees, the tree branches are terminated at the subsystem level, where they have been shown as events. The quantified data and logic for these subsystem events is taken from that derived from the subsystem fault trees as described in the previous section. To illustrate the phase relationships, phase 1 and 6 are discussed in detail. Phase 1 is for 'mission allocation and route optimisation', where firstly the laser navigation system (LNS) needs to correctly locate the AGV's position. Then, all routes for

Table 2 Part FMECA of AGV system

\begin{tabular}{|c|c|c|c|c|c|c|c|c|c|}
\hline Identity & Function & Sub-item & Failure mode & $\begin{array}{l}\text { Failure effect } \\
\text { Local effect }\end{array}$ & System effect & $\mathrm{S}$ & $\mathrm{F}$ & $\mathrm{D}$ & RPN \\
\hline \multirow[t]{4}{*}{$\begin{array}{l}\text { Laser navigation } \\
\text { system }\end{array}$} & $\begin{array}{l}\text { Locate correct } \\
\text { position. Send } \\
\text { and receive the } \\
\text { information of } \\
\text { location }\end{array}$ & GPS & Unit fails AGV & $\begin{array}{l}\text { Halt motion for } \\
\text { safety }\end{array}$ & $\begin{array}{l}\text { Routing and dis- } \\
\text { patching inhib- } \\
\text { ited }\end{array}$ & 3 & 3 & 4 & 36 \\
\hline & & Signal transmitter & $\begin{array}{l}\text { Unable to send } \\
\text { information of } \\
\text { location to the } \\
\text { central system }\end{array}$ & $\begin{array}{l}\text { Halt motion for } \\
\text { safety }\end{array}$ & $\begin{array}{l}\text { Routing and dis- } \\
\text { patching inhib- } \\
\text { ited }\end{array}$ & 3 & 3 & 4 & 36 \\
\hline & & Laser emitter & Unit fails & $\begin{array}{l}\text { Halt motion for } \\
\text { safety }\end{array}$ & $\begin{array}{l}\text { Route block; } \\
\text { AGV confliction }\end{array}$ & 3 & 3 & 4 & 36 \\
\hline & & Laser sensor & Unit fails & $\begin{array}{l}\text { Halt motion for } \\
\text { safety }\end{array}$ & Task fails & 2 & 3 & 4 & 24 \\
\hline
\end{tabular}




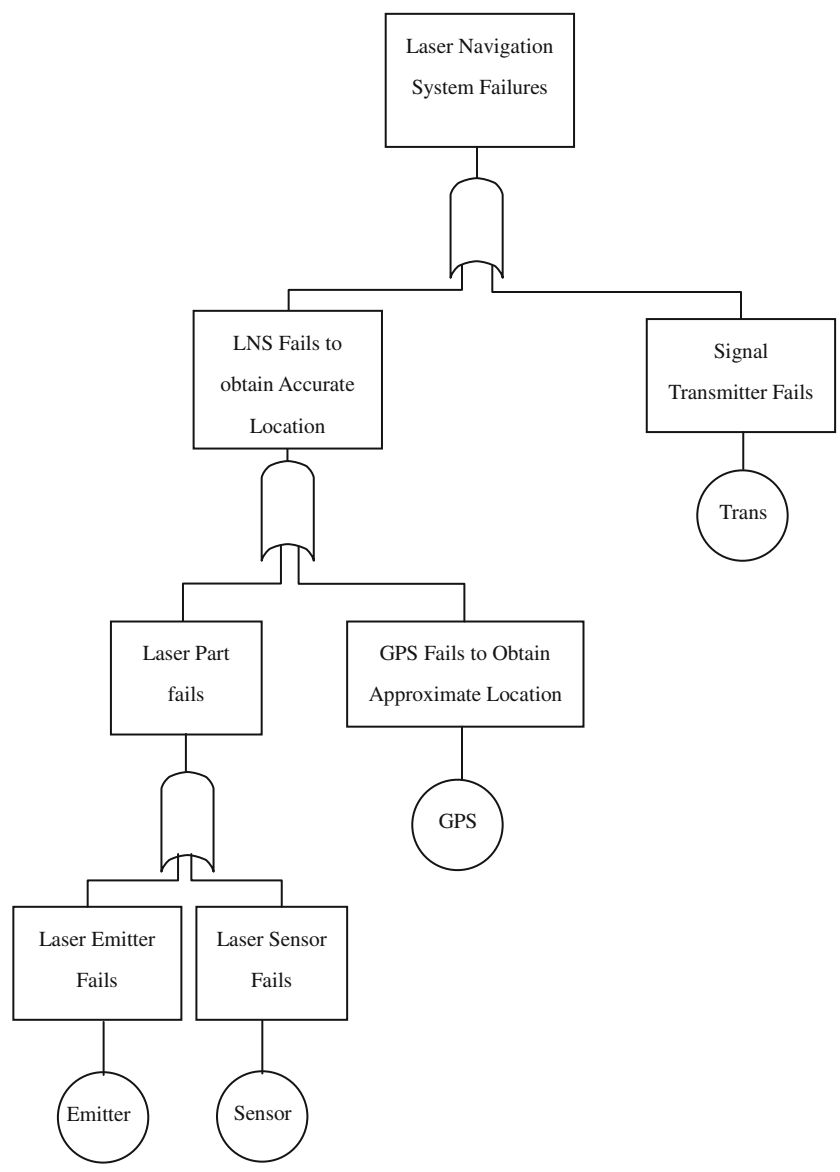

Fig. 3 Laser navigation subsystem level fault tree

completing the phase mission should be properly optimised via the AGV software control system (ASCS). The phase cannot be completed if either the LNS or ASCS fails to work properly. In throughout the phase. The fault tree for phase 1 is shown in Fig. 4.

In Phase 6, the fault tree for which is shown in Fig. 5, the AGV will travel from the storage back to the base. During this period, the ASCS will control the AGV to travel along the optimised route, the LNS will locate the AGV as it moves, the motor will drive the vehicle, the steering

Table 3 Calculated frequencies of the AGV subsystem failures

\begin{tabular}{ll}
\hline Subsystem & Failure rate (frequency/year) \\
\hline Drive unit & 1.5 \\
AGV software control system & 6 \\
Laser navigation system & 0.875 \\
Safety systems & 0.375 \\
Attachments & 2 \\
Battery & 1.25 \\
Brake system & 0.2 \\
Steering system & 0.25 \\
\hline
\end{tabular}

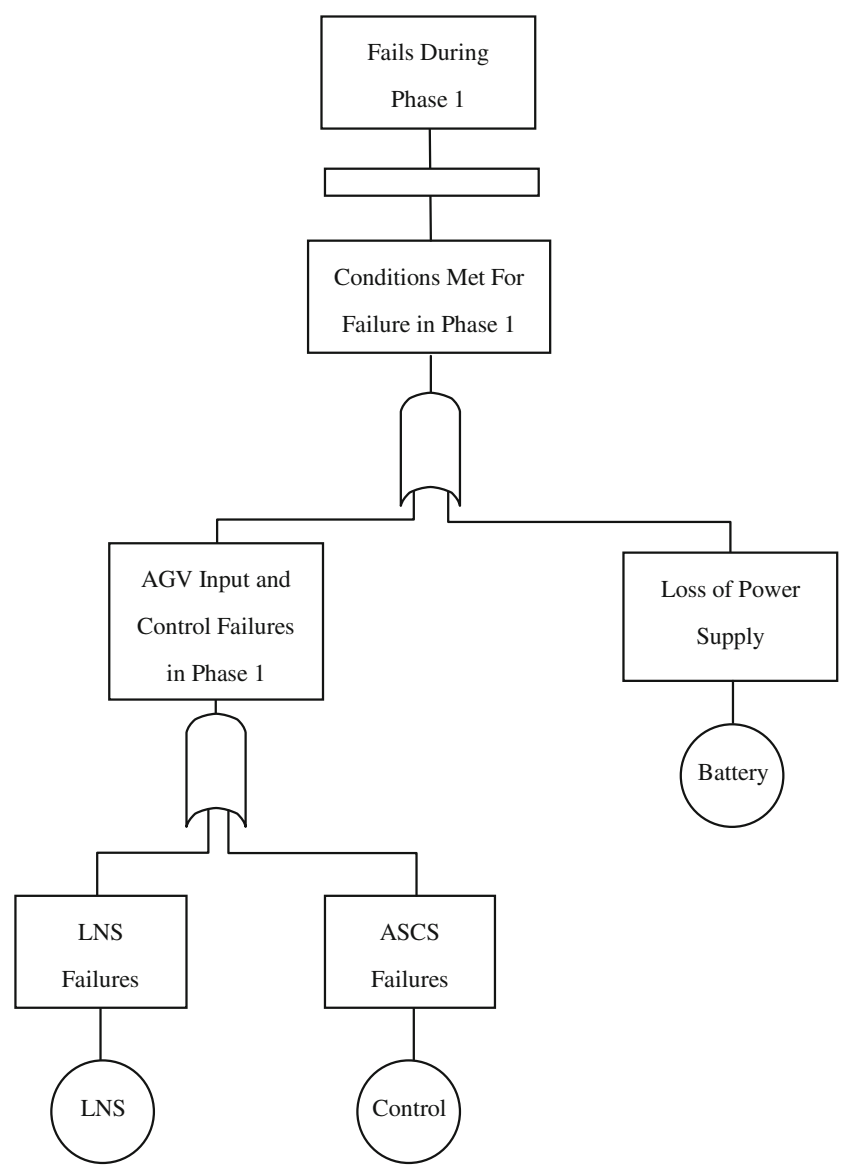

Fig. 4 Fault tree failure model for phase 1

system will enable vehicle turning, the safety system will perform an obstacle scan and the brake system will slow down the vehicle when turning and stop the vehicle if necessary to avoid collisions. Obviously, the success of phase 6 of the mission relies on the synchronous cooperation of all these subassemblies. The failure of any one of them can lead to the failure of the AGV during phase 6. In addition, phase 6 can be started only after all of the previous phases have been completed successfully. This is shown in Fig. 5 with the events from 'Functions Through Phase 1' to 'Functions Through Phase 5' and 'Conditions Met For Failure in Phase 6' joined under an 'AND' gate. The event 'Functions in phase 1' is the event 'Failure in Phase 1' under a 'NOT' gate, likewise for phase 2 to phase 5 . In general, the mission failure in phase $j+1$ is the combined result of successful phases 1 to $j$ and the system failure occurring in phase $j+1$ via an 'AND' gate and the 'NOT' gate is used to represent system success during phases 1 to $j$. Similar fault tree models for the remaining four phases have been created. In these models, all of the corresponding subsystems in each phase are required to work properly and synchronously as well and all previous phases must have been completed successfully. The later the phase in the mission the larger its fault 


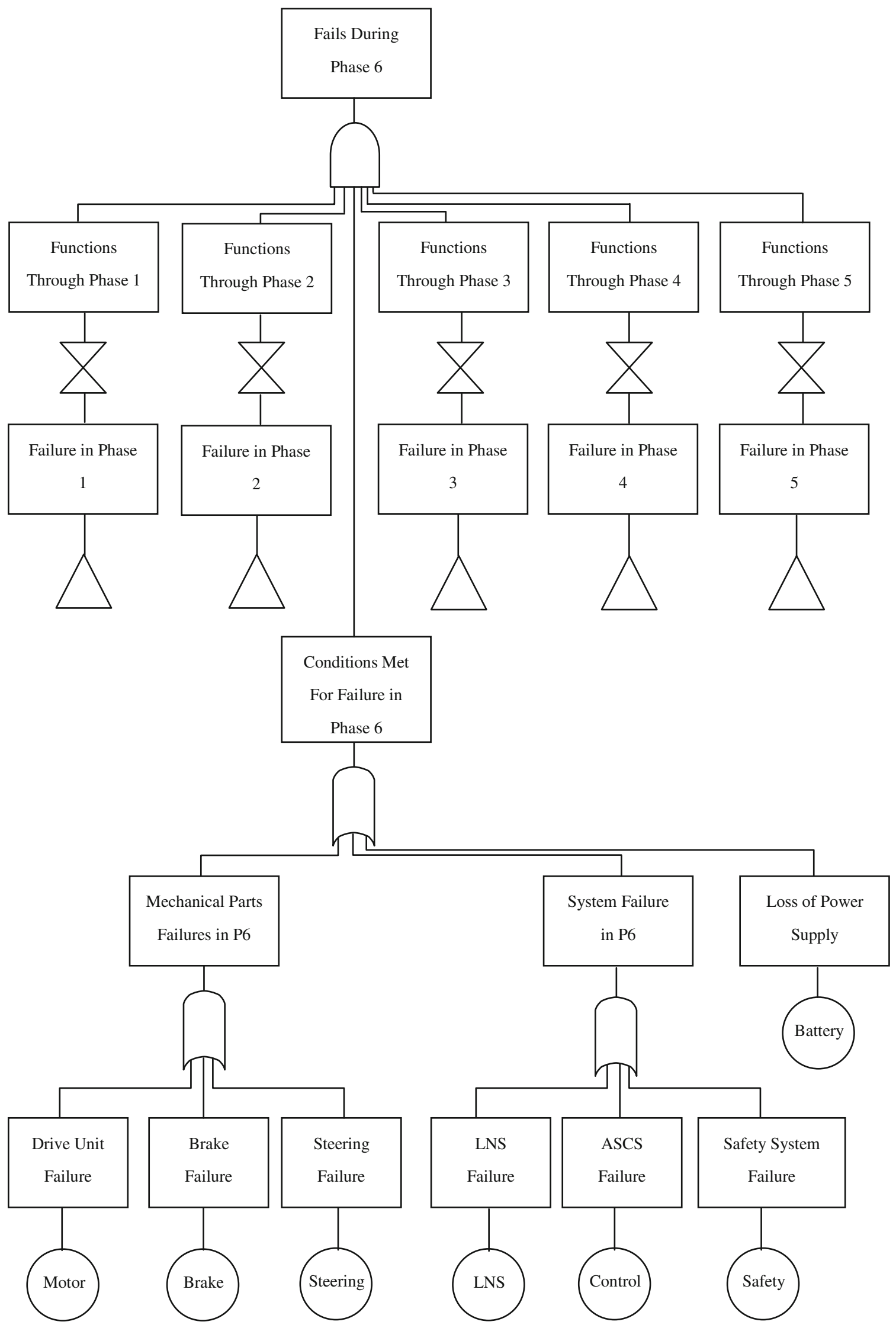

Fig. 5 Fault tree failure model for phase 6 
tree is, as the number of phases that must have been completed successfully increases. For example, the fault tree of phase 6 requires the successful completions of all previous five phases, this results in the tree containing 73 individual events and 44 gates. Using the fault trees developed, the AGV operation can be both qualitatively and quantitatively analysed at each phase.

The subsystem failures that lead to the different phase failures are listed in Table 4 and the phase failure probabilities obtained from analysing the fault trees are also shown in the table. As an example of how these results were obtained, consider phases 1 and 2. Due to the existence of NOT gates in the phase fault trees for failure during any phase after the first one, the fault trees are non-coherent. In this case, the occurrence of the system failure in the phase can be expressed using the prime implicants. The prime implicants are the minimal combination of component states that cause top event failure. For example, the prime implicants for failures within phase 1 and phase 2 , represented by $T_{1}$ and $T_{2}$, respectively, can be computed using the following expressions:

$$
\begin{aligned}
T_{1}= & \text { Failure in } P 1 \\
= & \text { ASCS }_{1}+\mathrm{LNS}_{1}+\text { Battery }_{1} \\
T_{2}= & (\text { Failure in } P 2) \cdot(\text { Success up to } P 2) \\
= & \left(\mathrm{DC}_{1,2}+\text { Brake }_{1,2}+\text { Steering }_{1,2}\right. \\
& + \text { ASCS }_{1,2}+\mathrm{LNS}_{1,2}+\mathrm{SS}_{1,2} \\
& \left.+ \text { Battery }_{1,2}\right) \cdot\left(\overline{\operatorname{ASCS}_{1}+\mathrm{LNS}_{1}+\text { Battery }_{1}}\right)
\end{aligned}
$$

Table 4 Subsystem failures that cause the failure at each phase

\begin{tabular}{lll}
\hline Phase & $\begin{array}{l}\text { Subsystem failures causing phase } \\
\text { failure at each phase }\end{array}$ & Phase unreliability \\
\hline 1 & $\begin{array}{l}\text { ASCS; LNS; battery } \\
\text { Drive unit (DC); brake system; }\end{array}$ & 0.00001855 \\
steering system; ASCS; LNS; & \\
safety system (SS); battery \\
Attachments; brake system; & 0.00024386 \\
& $\begin{array}{l}\text { ASCS; safety system; battery; } \\
\text { Drive unit; ASCS; LNS; safety }\end{array}$ & 0.00021915 \\
& $\begin{array}{l}\text { system; attachments; battery; } \\
\text { brake system; steering system }\end{array}$ & \\
& $\begin{array}{l}\text { Attachments; brake system; } \\
\text { ASCS; safety system; battery; }\end{array}$ & 0.00002243 \\
6 & $\begin{array}{l}\text { Drive unit; ASCS; LNS; safety } \\
\text { system; battery; brake system; }\end{array}$ & 0.00012527 \\
steering system
\end{tabular}

where subscript 1 denotes failure in phase 1 and subscript 1,2 denotes failure in any phase from 1 to 2 . Expressions $T_{1}$ and $T_{2}$ are obtained from the fault trees for phases 1 and 2, respectively. As can be seen from Fig. 4 for $T_{1}$. As the number of components and phases increases the derivation of prime implicants also becomes more complex. Once the prime implicants are known, the calculation of unreliability in phase 1 and phase 2 can be conducted using the inclusion-exclusion principle as shown in [25]. For example, the probability of failure up to the end of phase $1, Q_{1}$, can be calculated as

$$
\begin{aligned}
Q_{1}= & \operatorname{Pr}\left(\mathrm{ASCS}_{1}\right)+\operatorname{Pr}\left(\mathrm{LNS}_{1}\right)+\operatorname{Pr}\left(\text { Battery }_{1}\right) \\
& -\operatorname{Pr}\left(\mathrm{ASCS}_{1} \cdot \text { Battery }_{1}\right) \\
& -\operatorname{Pr}\left(\mathrm{ASCS}_{1} \cdot \text { Battery }_{1}\right) \\
& -\operatorname{Pr}\left(\mathrm{LNS}_{1} \cdot \text { Battery }_{1}\right) \\
& +\operatorname{Pr}\left(\mathrm{ASCS}_{1} \cdot \mathrm{LNS}_{1} \cdot \text { Battery }_{1}\right) \\
= & q_{\mathrm{ASCS}_{1}}+q_{\mathrm{LNS}_{1}}+q_{\mathrm{Batter}_{1}} \\
& -q_{\mathrm{ASCS}_{1}} \cdot q_{\mathrm{LNS}_{1}} \\
& -q_{\mathrm{ASCS}_{1}} \cdot q_{\mathrm{Batter}_{1}} \\
& -q_{\mathrm{LNS}_{1}} \cdot q_{\mathrm{Battery}_{1}} \\
& +q_{\mathrm{ASCS}_{1}} \cdot q_{\mathrm{LNS}_{1}} \cdot q_{\mathrm{Batter}_{1}}
\end{aligned}
$$

The probability of failure of basic event $A$ in all phases from $i$ to $j$ (i.e. $q_{A_{i, j}}$ ) can be calculated using Eq. 4:

$q_{A_{i, j}}=e^{-\lambda_{A} t_{i-1}}-e^{-\lambda_{A} t_{j}}$

where $\lambda_{A}$ refers to the failure rate of a basic event $A, t_{j}$ is the length of phase $j$. The phase $j\left(P_{j}\right)$ unreliability or failure probability can be determined using:

$$
\begin{aligned}
\operatorname{Pr}\left(P_{j} \text { Failure }\right) & =1-\frac{\text { Success up to end of } P_{j}}{\text { Success up to end of } P_{j-1}} \\
& =1-\frac{1-Q_{1, j}}{1-Q_{1, j-1}}
\end{aligned}
$$

where $Q_{1, j}$ refers to the unreliability in all phases from 1 to $j$. It should be noticed that the probability of failure is calculated using the exponential distribution as it is assumed that the AGV fails when the components are in their useful life periods.

For more complex systems or missions especially involving dependencies of the components, the derivation of the prime implicants for reliability calculation becomes increasingly complex. Therefore, using FTA for mission analysis requires extensive processing power, and therefore the PN technique has been investigated. 


\section{Mission level Petri net model generation}

Petri net analysis has been adopted to investigate the reliability of the mission, initially the Petri net model itself is generated, which involves a three tiered approach with the subsystem Petri nets (SPN) feeding into the phase Petri net (PPN), which feeds the master mission Petri net (MPN).

\subsection{Subsystem Petri net(SPN)}

Given the modularity of the subsystems, this has been chosen as the starting point for the lower tier of Petri net models, referred to as the subsystem Petri net (SPN) model, as shown in Fig. 6.

The subsystem labels $1-8$ correspond to the subsystem failure modes listed in Table 3. These PN models are used to model the health states of each of the AGV subsystems. For more complex architectures, this tier can have a preceding tier represented by component models. As the mission has been modelled as a MFOP, the repair of subsystems will not be considered in this study. Therefore, the SPN will show only two kinds of health state, i.e. 'subsystem up' and 'subsystem down'. Once a subsystem fails after working for a certain period of time, the token in the 'subsystem up' place will be transferred to the 'subsystem down' place. The time for this failure transition can be computed by using the random sampling and exponential distribution method, using the component failure rate data from the subsystem modelling given in Table 3 [29].

The information about subsystem failures can then be fed into the phase Petri net (PPN) models, using linking-arcs, which are the dashed lines in Fig. 6.

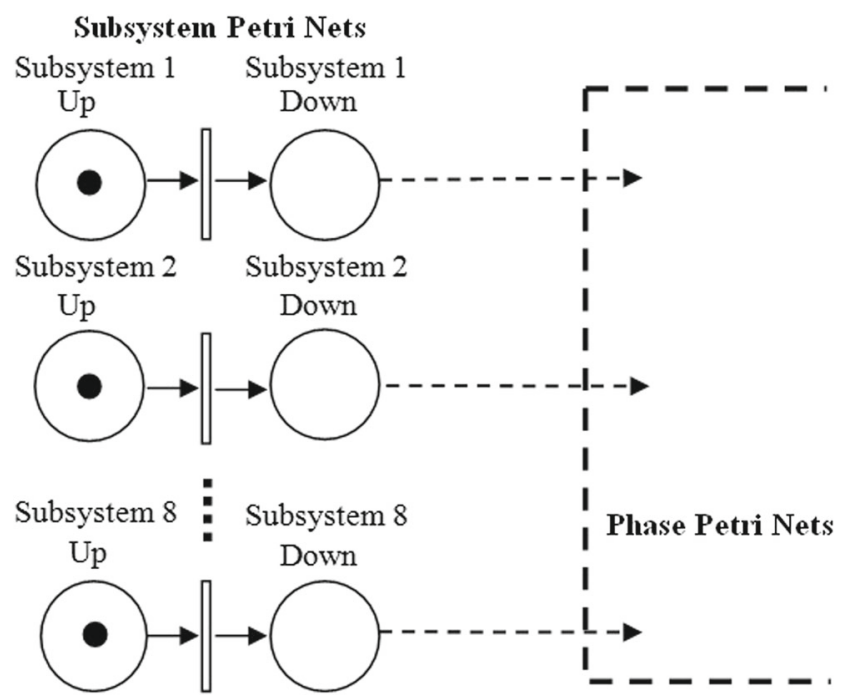

Fig. 6 Subsystem Petri net
LNS Down

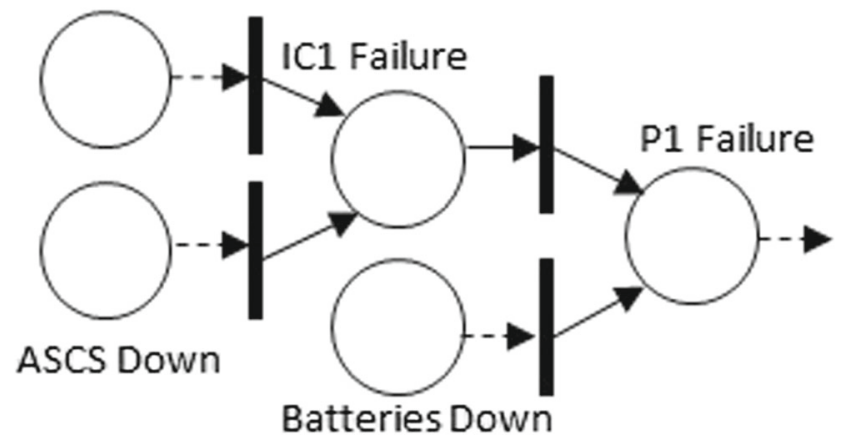

Fig. 7 Phase Petri net for phase 1

\subsection{Phase Petri net (PPN)}

The PPN presents the interrelated subsystem failure mechanisms that correspond to failure in the phase. The logic used for implementing FTA can be directly applied when constructing the PPNs. For this reason, the PPN's constructed for phases 1 and 6 , which relate to the fault trees in Figs. 4 and 5, are shown in Figs. 7 and 8 to ease understanding. The transitions are instantaneous and are represented by solid rectangular bars. Comparing the fault tree for phase 6 in Fig. 5 and the corresponding PPN in Fig. 8, it can be seen

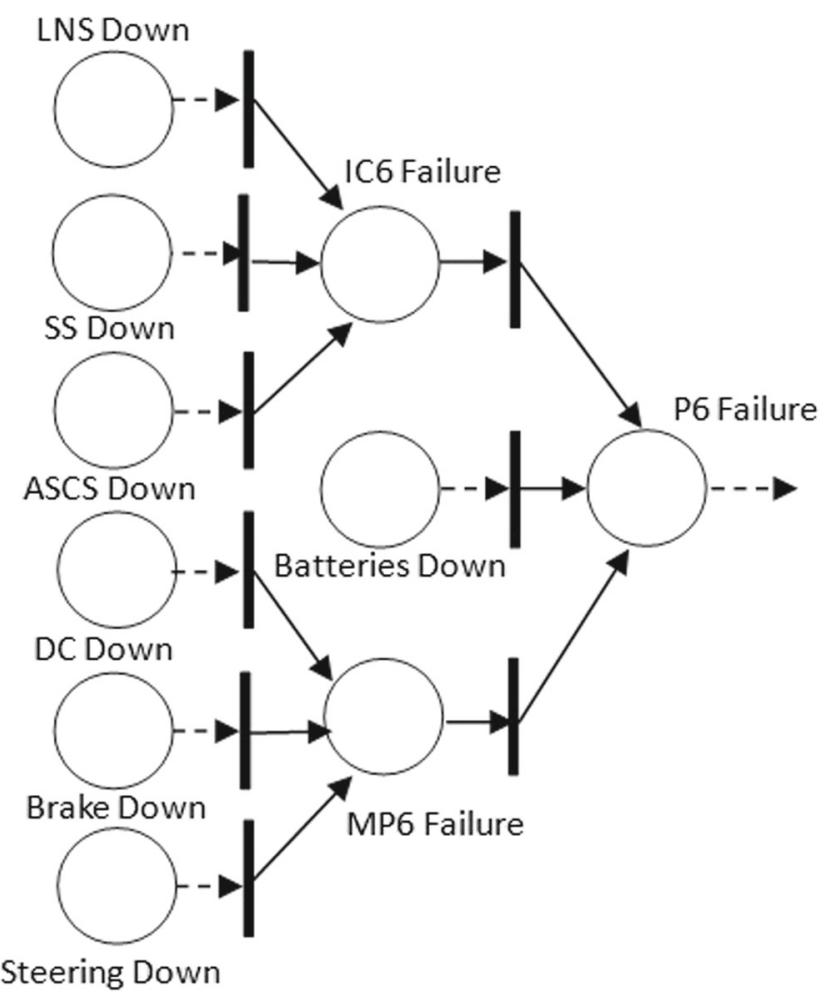

Fig. 8 Phase Petri net for phase 6 
that there is no place in the PN corresponding to the events for functioning through the previous phases in the fault tree. This is dealt with in the master Petri net described in the following section. Tokens are absent from all places in Figs. 7 and 8, illustrating that the whole AGV system is in a good health condition. In other words, the presence of a token in a place will mean the failure of either a subsystem or a phase.

\subsection{Master Petri net (MPN)}

The MPN is used to govern the change of phases from the beginning of the mission, phase 1 , to the successful completion of the whole mission, at the end of phase 6 . Figure 9 shows the structure of the MPN, where a token in the phase place is used to indicate the phase that the AGV is operating in. The system failure happening in each phase, i.e. the top event of the PPN for that phase, will directly result in the failure of the whole mission. Hence, if the AGV is operating in phase $i$ so that a token resides in place 'phase i' and the AGV fails in that phase, so a token is in place 'Pi failure' then a token will be transferred to the system failure place, so that the mission fails. The switching time of transition between two neighbouring phase places is the length of the preceding phase. Likewise, the switching time of transition between phase 6 and mission finish is the length of phase 6 . If the AGV completes all six phases without a phase failure, then a token will be placed in the 'simulation success' place.

\section{Simulation and results}

\subsection{Simulation model}

In order to calculate results about how reliable the operation of the AGV mission is, the PN model has been embedded

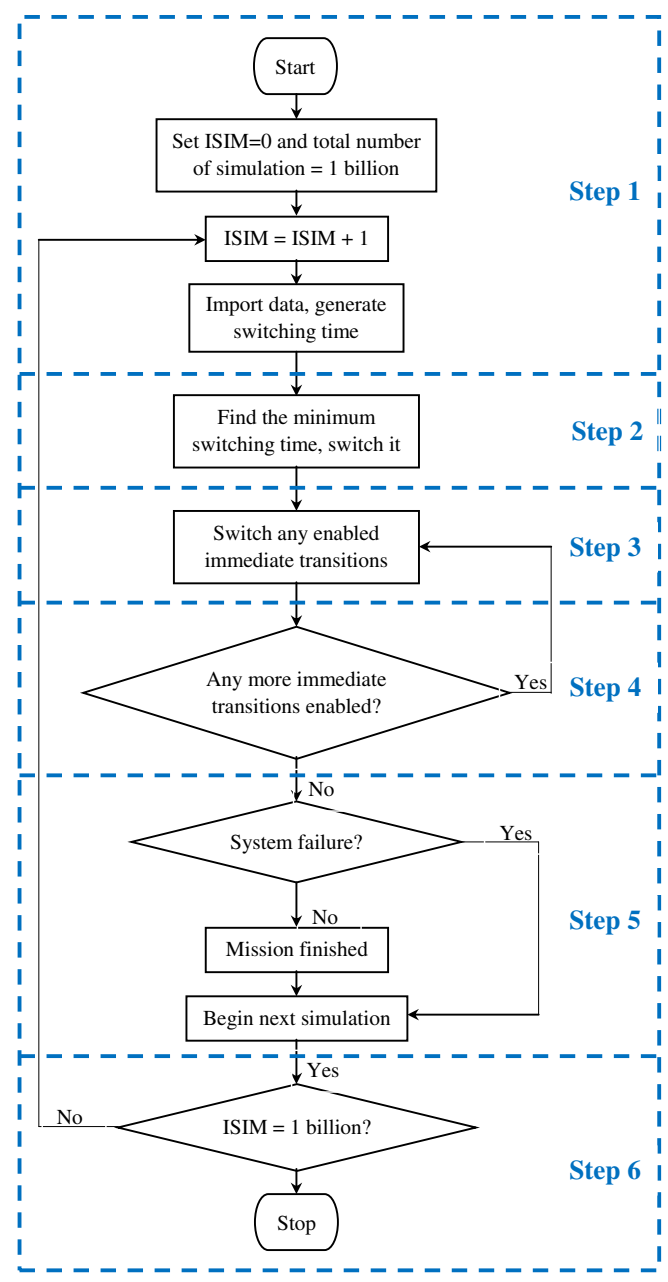

Fig. 10 Simulation flowchart

in a simulation model. The failure rates of all AGV subsystems and the phase lengths are used as inputs of the

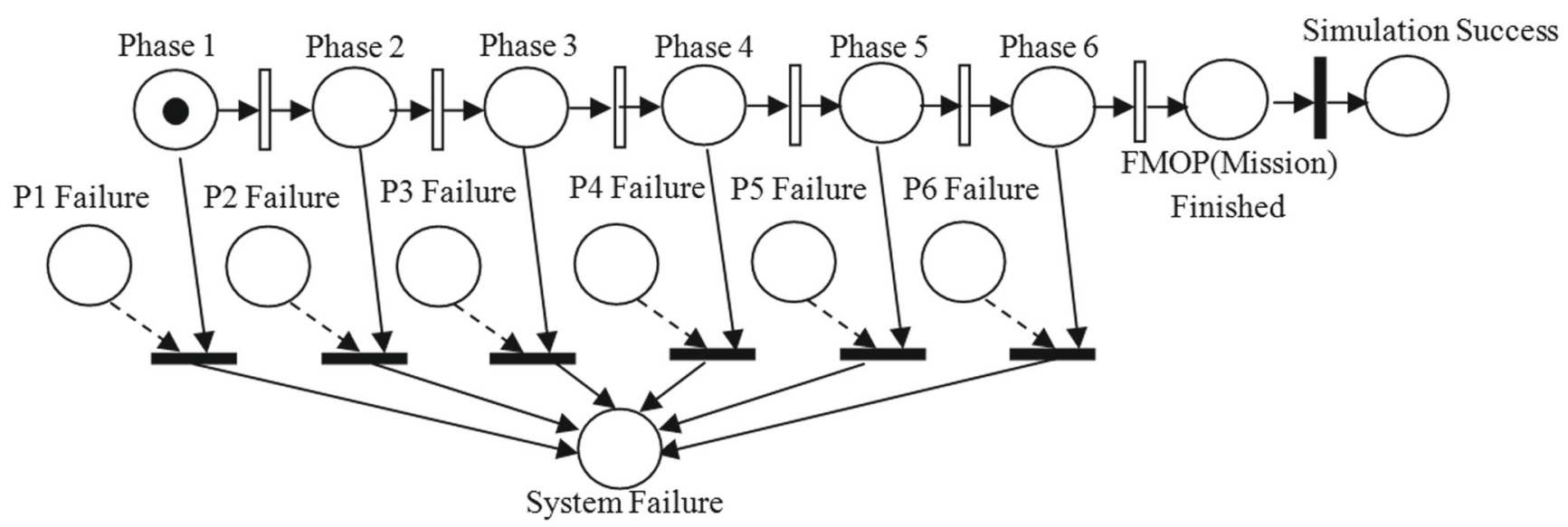

Fig. 9 Master Petri net 
PN simulation. Then, the simulation can be programmed by using the following steps (as illustrated in Fig. 10):

Step 1: Import the phase lengths into the MPN and in parallel, generate the switching time of the transitions of each subsystem in the SPN's by using the randomly sampling and exponential distribution method;

Step 2: Find the transition with the minimum switching time and then switch it;

Step 3: Search through the immediate transitions that are directly connected to the present place. If any are found enabled, switch them;

Step 4: Repeat Step 3 until no more immediate transitions are enabled;

Step 5: Test for any of the following conditions and log them: (a) if system has failed, begin next simulation; (b) if mission has completed, begin next simulation.

Step 6: Iterate the above simulation for $\mathrm{n}$ times based on the assumption that the reliability of the AGV system can be obtained by repeating the simulation for a sufficient number of times.

\subsection{Results and validation}

Embedding the PN into a simulation, the phase unreliability and mission reliability have been calculated. The results obtained are shown in Table 5 (columns 1-5). In order to ensure a good convergence of the computing result, one billion simulations have been performed in the process of this calculation. The results have been validated by using the FTA method to calculate the phase and mission reliability (results shown in columns 6 and 7 of Table 5). The comparison shows that the simulation results obtained from the PN method are very close to the analytical solutions derived from FTA. The simulation errors of both the unreliability

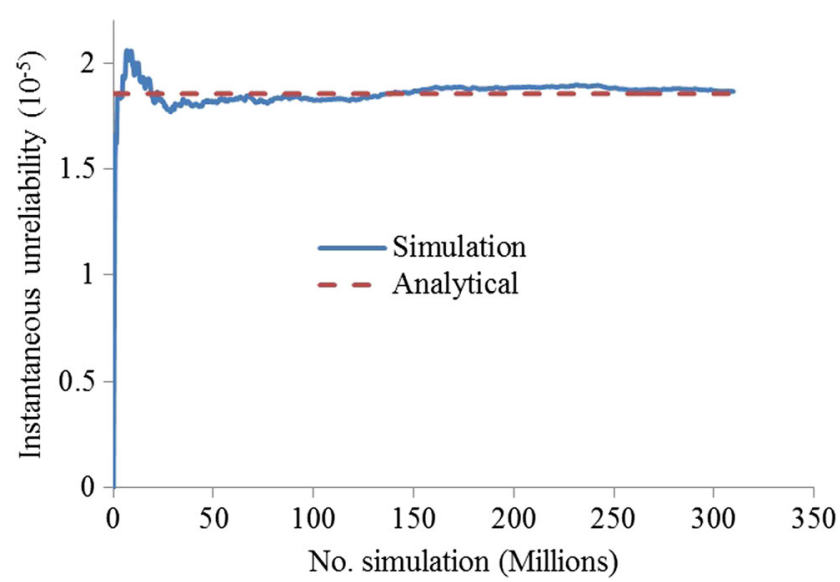

Fig. 11 Convergence of phase 1 unreliability

of each phase and the mission reliability at the end of each phase are below $1 \%$.

Considering the convergence of the results, Fig. 11 shows the comparison of the analytical and simulated solution for phase 1 as the number of simulations increases. As can be seen in the figure the value of the unreliability of phase 1 obtained from the simulation has converged to the analytical result after performing approximately 100 million simulations. Similar results were found for the other phases. Hence, performing 1 billion simulations is sufficient to guarantee the reliability of the calculated result.

From the results presented in Table 5, it is found that phase 2 'dispatch to station' and phase 4 'travelling to storage' show the largest phase unreliability values. This means that the AGV is more likely to fail when it is undertaking the tasks of these two phases. Additionally, it is found that the mission reliability at the end of the sixth phase is 0.999297 , which is based on the success of all six phases. Hence, this

Table 5 PN simulation results

\begin{tabular}{lllllll}
\hline Phase & Phase failures & Phases started & $\begin{array}{l}\text { Phase } \\
\text { unreliability }\end{array}$ & $\begin{array}{l}\text { Mission reliabil- } \\
\text { ity at phase end }\end{array}$ & $\begin{array}{l}\text { Phase } \\
\text { (FT) }\end{array}$ & $\begin{array}{l}\text { Mnreliability } \\
\text { Mission reliabil- } \\
\text { ity at phase end } \\
\text { (FT) }\end{array}$ \\
\hline 1 & 18449 & 1000000000 & 0.00001845 & 0.999982 & 0.00001855 & 0.999981 \\
2 & 244863 & 999981551 & 0.00024486 & 0.999737 & 0.00024386 & 0.999738 \\
3 & 72843 & 999736688 & 0.00007286 & 0.999664 & 0.00007266 & 0.999665 \\
4 & 218911 & 999663845 & 0.00021898 & 0.999445 & 0.00021915 & 0.999446 \\
5 & 22488 & 999444934 & 0.00002250 & 0.999422 & 0.00002243 & 0.999423 \\
6 & 125509 & 999422446 & 0.00012558 & 0.999297 & 0.00012527 & 0.999298
\end{tabular}


Table 6 Phase failures during 600000 MFOP simulations

\begin{tabular}{lllllllll}
\hline \multirow{2}{*}{ Phase } & Phase started & \multicolumn{2}{l}{ Failures in } & \multicolumn{3}{c}{ Total failure Phase unreliability } \\
\cline { 3 - 6 } & & & Mission 1 & Mission 2 & $\ldots$ & Mission 500 & \\
\hline 1 & 251755556 & 7 & 12 & $\ldots$ & 7 & 4667 & 0.00001854 \\
2 & 251750889 & 162 & 138 & $\ldots$ & 101 & 61589 & 0.00024464 \\
3 & 251689300 & 41 & 54 & $\ldots$ & 47 & 24193 & 0.00009612 \\
4 & 251665107 & 115 & 132 & $\ldots$ & 82 & 55059 & 0.00021878 \\
5 & 251610048 & 11 & 9 & $\ldots$ & 10 & 5654 & 0.00002247 \\
6 & 251604394 & 69 & 75 & $\ldots$ & 66 & 31552 & 0.00012540 \\
\hline
\end{tabular}

value also indicates the overall reliability of the AGV in accomplishing the whole mission. This means that the AGV has a greater than $99 \%$ chance of successfully completing the mission. For this reason, it can be concluded that the AGV considered here is a very reliable material distribution vehicle in the warehouse. However these are results for only one mission and it is expected that the AGV will perform numerous missions and hence its reliability as the number of missions increases is of interest. As can be seen from Table 5 , the mission reliability at the end of each phase decreases gradually against the number of phases that the AGV has successfully completed. This suggests that without maintenance, the more missions are completed, the more unreliable the AGV system will be.

Hence, the PN model has been run to simulate the AGV system performing a number of continuous consecutive missions without maintenance. The results for 600000 individual MFOP, each of them containing 500 consecutive missions, are shown in Table 6, where the unreliability for each phase is shown. The number of simulations, failures and reliabilities for each mission and overall MFOP are shown in Table 7. As can be seen, the reliability of the AGV for completing the MFOP with 500 missions is 0.69547667. Obviously, such a method is very helpful in determining the

Table 7 MFOP and mission failure results

\begin{tabular}{llll}
\hline MFOP/mission & Starts & Failures & Reliability \\
\hline Mission 1 & 600000 & 405 & 0.99932500 \\
Mission 2 & 599595 & 420 & 0.99929953 \\
$\vdots$ & $\vdots$ & $\vdots$ & $\vdots$ \\
Mission 500 & 417599 & 313 & 0.99925048 \\
MFOP & 600000 & 182714 & 0.69547667 \\
\hline
\end{tabular}

optimal inspection interval for performing the maintenance of the AGV.

Following this logic, the reliability of the MFOPs with different number of consecutive missions is calculated via simulation. The simulation results are shown in Fig. 12, from which it is clearly observed that the reliability of the $\mathrm{AGV}$ is reduced with the increasing number of missions. Accordingly, through observing such a decreasing tendency of the reliability of the AGV against the number of missions, the optimal inspection and maintenance time can be readily estimated to ensure the reliability of the AGV can be above a desired level.

The research documented in this paper demonstrates that the PN method can be used for AGV reliability assessment. It can easily be adapted to cater for varying complexities of the subsystems and number of AGVs, where coloured Petri nets can be used. In contrast to the fault tree approach, the PN simulation method does not require finding a complex qualitative solution. Hence, it is a promising time saving and effective approach that can be widely used in the future to deal with the reliability problems existing in complex systems.

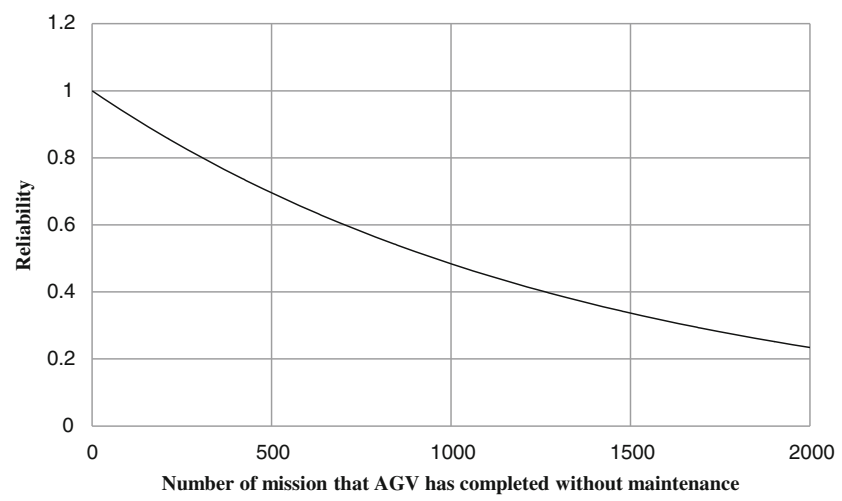

Fig. 12 Reliability vs. mission number 


\section{Conclusions}

In order to develop an efficient and reliable approach to assessing the reliability of AGVs, the PN method is adopted in this paper to calculate the mission and phase reliability of a typical AGV transport system. Due to the size of the system considered and the mission profile, FTA was also performed and the results compared with those obtained using PN's. Agreement was seen to be good. FTA is limited in its use for complex systems as dependencies cannot be accurately modelled. It is also increasingly complex and slow as the number of phases in a mission increases. PNs have none of these limitations and hence can easily be extended to more complex systems and missions with numerous phases. Through this research, the following conclusions can be drawn: (1) the PN method has been demonstrated to be an effective approach for conducting AGV system mission reliability assessment providing the capability to make informed decisions regarding the acceptability of AGV mission performance; (2) the results have suggested that the AGV is more likely to fail when completing the phase 'dispatch to station' and the phase 'travelling to storage'. It is worthy to note that such a judgement is made only based on the assumptions given in Tables 1 and 3. In reality, the judgement result would be different, depending on the environmental, loading and operational conditions of the AGVs. These are easily adapted within the modelling approach thus enabling more complex AGV systems to be modelled; (3) by using the PN method, the influence of maintenance and the optimal time for maintenance can be evaluated. (4) The PN method is able to account for dependencies which may occur within subsystem and across mission phases enabling reliable capability evaluations for AGV systems of the future with expanding fleets.

It is worth mentioning that if the AGV system is relatively simple with no dependencies and the mission does not involve multiple tasks then FTA is applicable. However, if any complexities are involved or maintenance needs to be considered then, as shown here, combining the FTA with PN simulation is an efficient approach.

Future work includes expanding the model to include the routing problem once failure of an AGV occurs in a multi-AGV system. The mission and route will be analysed simultaneously. Also, it is planned that the proposed method will be validated in a real AGV system through the collaboration with relevant industry partners.

Acknowledgements The work reported in this paper aligns to the working being researched as part of the EPSRC grant EP/K014137/1. The authors would like to extend thanks to Dr Cunjia Liu at Loughborough University and Mr Dave Berridge at Automated Materials Handling Systems Association for their kind help in preparing this paper.
Open Access This article is distributed under the terms of the Creative Commons Attribution 4.0 International License (http:// creativecommons.org/licenses/by/4.0/), which permits unrestricted use, distribution, and reproduction in any medium, provided you give appropriate credit to the original author(s) and the source, provide a link to the Creative Commons license, and indicate if changes were made.

\section{References}

1. Vis IF (2006) Survey of research in the design and control of automated guided vehicle. Eur J Oper Res 170(3):677709

2. Tuan LA, Koster De MMD (2006) A review of design and control of automated guided vehicle systems. Eur J Oper Res 171(1):123

3. Miljković Z, Vuković N, Mitić M, Babić B (2013) New hybrid vision-based control approach for automated guided vehicles. Int J Adv Manuf Technol 66(1):231-249

4. Bhattacharya R, Bandyopadhyay S (2010) An improved strategy to solve shop deadlock problem based on the study on existing benchmark recovery strategies. Int J Adv Manuf Technol 47:351364

5. Giuseppe C, Fabiano M, Liotta G (2013) A network flow based heuristic approach for optimising AGV. J Intell Manuf 24:405419

6. Salehipour A, Sepehri MM (2014) Optimal location of workstations in tandem automated-guided vehicle systems. Int J Adv Manuf Technol 72(9):1429-1438

7. Mallikarjuna K, Veeranna V, Reddy K (2016) A new metaheuristics for optimum design of loop layout in flexible manufacturing system with integrated scheduling. Int J Adv Manuf Technol 84(9):1841-1860

8. Wu NQ, Zhou MC (2007) Shortest routing of bidirectional automated guided vehicles avoiding deadlock and blocking. IEEE/ASME Trans Mechatron 12(1):63-72

9. Nishi T, Tanaka Y (2012) Petri net decomposition approach for dispatching and conflict-free routing of bidirectional automated guided vehicle systems. IEEE Trans Syst Man, Cybern 42(5):1230-1243

10. Rifai AP, Dawal SZMd, Zuhdi A, Aoyama H, Case K. (2016) Re-entrant FMS scheduling in loop layout with consideration of multi loading-unloading stations and shortcuts. Int J Adv Manuf Technol 82(9):1527-1545

11. Vivaldini K, Rocha LF., Martarelli NJ, Becker M, Paulo Moreira A (2016) Integrated tasks assignment and routing for the estimation of the optimal number of AGVS. Int J Adv Manuf Technol 82(1):719-736

12. Abdelmaguid TF, Nassef AO (2010) A constructive heuristic for the integrated scheduling of machines and multiple-load material handling equipment in job shops. Int $\mathbf{J}$ Adv Manuf Technol 46(9):1239-1251

13. Hamzheei M, Farahani RZ, Rashidi-Bajgan H (2013) An ant colony-based algorithm for finding the shortest bidirectional path for automated guided vehicles in a block layout. Int J Adv Manuf Technol 64(1):399-409

14. Trenkle A, Seibold Z, Stoll T (2013) Safety requirements and safety functions for decentralized controlled autonomous systems. In: XXIV International Conference on Information, Communication and Automation Technologies (ICAT)

15. Ebben M (2001) Logistic Control In Automated Transportation Networks. Ph.D. thesis, University of Twente 
16. Fazlollahtabar H, Saidi-Mehrabad M (2013) Optimising a multiobjective reliability assessment in multiple AGV manufacturing system. Int J Serv Oper Manag 3:352-372

17. Tavana M, Fazlollahtabar H, Hassanzadeh R (2014) A bi-objective stochastic programming model for optimising automated material handling systems with reliability considerations. Int J Prod Res 52(19):5597-5610

18. Duran DR, Robinson E, Kornecki AJ, Zalewski J (2013) Safety analysis of autonomous ground vehicle optical systems: Bayesian Belief Networks Approach Computer Science and Information Systems (FedCSIS), Federated Conference

19. XYang Wu, XYue Wu (2015) Extended object-oriented Petri net model for mission reliability simulation of repairable PMS with common cause failures. Reliab Eng Syst Saf 130:109-119

20. Le B, Andrews JD (2014) Modelling wind turbine degradation and maintenance. Wind Energy 19:571-591

21. Luo JL, Ni HJ, Zhou MC (2015) Control program design for automated guided vehicle systems via Petri nets. IEEE Trans Syst Man, Cybern: Syst 45(1):44-55
22. Nishi T, Maeno R (2010) Petri net decomposition approach to optimization of route planning problems for AGV systems. IEEE Trans Autom Sci Eng 7(3):523-537

23. Petri CA (1962) Kommunikation mit automaten, PhD thesis

24. Mura I, Bondavalli A (2001) Markov regenerative stochastic petri nets to model and evaluate phased mission systems dependability. IEEE Trans Comput 50(12):1337-1351

25. Chew SP, Dunnett SJ, Andrews JD (2008) Phased mission modelling of systems with maintenance-free operating periods using simulated Petri nets. Reliab Eng Syst Saf 93(7):980-994

26. MacLeod EN, Chiarella M (1993) Navigation and control breakthrough for automated mobility. Proceedings of the SPIE mobile robotics VIII, Boston MA

27. Division M (1998) Design for success Ultra Reliable Aircraft project. Design 212:371-378

28. FMD-97 (1997) Failure Mode/Mechanism Distributions, Reliability Analysis Center

29. Andrews JD, Moss TR (2002) Reliability and risk assessment, 2nd edn. Professional Engineering Publishing 